\title{
Tacrolimus Induces Glomerular Injury via Endothelial Dysfunction Caused by Reactive Oxygen Species and Inflammatory Change
}

\author{
Kengo Kidokoro Minoru Satoh Hajime Nagasu Takeo Sakuta \\ Atsunori Kuwabara Daisuke Yorimitsu Yuko Nishi Naruya Tomita \\ Tamaki Sasaki Naoki Kashihara \\ Department of Nephrology and Hypertension, Kawasaki Medical School Kurashiki, Okayama, Japan
}

\section{Key Words}

Angiotensin II receptor blocker • Nephrotoxicity $\cdot \mathrm{NAD}(\mathrm{P}) \mathrm{H}$ oxidase $\cdot$ Oxidative stress $\cdot$ Tacrolimus

\begin{abstract}
Background/Aims: The immunosuppressive drug tacrolimus (FK506) is used clinically to reduce the rejection rate in patients with kidney transplantation; however, the resultant nephrotoxicity remains a serious problem. In the present study we attempted to elucidate the mechanisms of glomerular injury induced by FK506 and the renoprotective effects of the angiotensin II receptor blocker telmisartan. Methods: Seven-week-old male Wistar rats were divided into three groups: vehicle group, FK506 group, and FK506 + telmisartan group. After 8 weeks, we assessed kidney function and renal morphological changes including oxidative stress. We also assessed the effect of FK506 in human glomerular endothelial cells (hGECs) with regard to reactive oxygen species (ROS). Results: FK506 induced ROS production via activation of $\mathrm{NAD}(\mathrm{P}) \mathrm{H}$ oxidase in the glomeruli. Expression of ICAM mRNA was increased in glomeruli from the FK506 group. These effects resulted in macrophage infiltration into the glomeruli. FK506 directly promoted NAD(P)H oxidase activity and accelerated production of ROS in hGECs. Conversely, cotreatment with telmisartan inhibited both
\end{abstract}

(C) 2012 S. Karger AG, Basel

$1420-4096 / 12 / 0356-0549 \$ 38.00 / 0$

Fax +41613061234

E-Mail karger@karger.ch

www.karger.com
Accessible online at: www.karger.com/kbr
$\mathrm{NAD}(\mathrm{P}) \mathrm{H}$ oxidase activity and production of ROS. Conclusion: These findings suggest that glomerular injury resulting from FK506 is caused by oxidative stress mediated by activation of $\mathrm{NAD}(\mathrm{P}) \mathrm{H}$ oxidase and that telmisartan exerts a renoprotective effect via antioxidative activity.

Copyright $\odot 2012$ S. Karger AG, Basel

\section{Introduction}

The calcineurin inhibitor (CNI) tacrolimus (FK506) is used clinically to reduce the rejection rate in patients with kidney transplantation. Recently, CNIs have become pivotal for the prevention of allograft rejection [1]. FK506 is more potent than cyclosporine $\mathrm{A}$ in the suppression of $\mathrm{T}$ cell activation and is often used when cyclosporine $A$ is not effective against acute rejection or when intolerance is an issue $[2,3]$. However, the nephrotoxicity resulting from FK506 remains a serious problem.

Acute CNI nephrotoxicity is considered a functional impairment of renal hemodynamics caused by vasoconstriction of afferent arterioles in response to use of this class of drug [4]. Thus, the acute form of nephrotoxicity is reversible. However, long-term use of $\mathrm{CNI}$ is associated with irreversible renal dysfunction attributable to progressive forms of tubulointerstitial and glomerular inju- 
ry. The histological changes typically observed in chronic CNI nephrotoxicity cases include arteriolar hyalinosis, tubular atrophy, interstitial fibrosis, thickening and fibrosis of the Bowman's capsule, and focal or global glomerular sclerosis [5]. Chronic tacrolimus nephropathy is also associated with arteriolopathy and narrowing of the arteriolar lumen, which are major contributors to the development of interstitial fibrosis, tubular atrophy, and glomerular sclerosis [6].

Multiple factors have been reported to mediate the pathogenic mechanisms underlying CNI nephrotoxicity and include increased production of vasoconstriction factors, such as endothelin or thromboxane, and a decrease in vasodilation factors like prostacyclin, prostaglandin $\mathrm{E}_{2}$, and nitric oxide. Activation of the renin-angiotensin system (RAS) has also been reported to mediate CNI nephrotoxicity [7-9]. In addition, several reports indicate that CNIs increase superoxide production, likely through vasoconstriction-associated hypoxia and/or direct effects $[10,11]$.

We have shown that increased production of reactive oxygen species (ROS) leads not only to the alteration of renal hemodynamics, but also to the development and progression of glomerular injury in various disease models [12]. In addition, the RAS is implicated in the generation of ROS through activation of $\mathrm{NAD}(\mathrm{P}) \mathrm{H}$ oxidase. We also clarified that angiotensin receptor blockers (ARB) ameliorate glomerular injury by suppression of oxidative stress [13]. CNIs have been indicated to promote generation of ROS in a number of cell types including mesangial cells $[14,15]$. Based on these findings, we hypothesize that FK506 enhances glomerular oxidative stress resulting in glomerular injury. We have attempted to elucidate the mechanisms of glomerulopathy induced by FK506 and the renoprotective effects of the ARB telmisartan.

\section{Materials and Methods}

\section{Experimental Protocol and Tissue Preparation}

The experimental protocol (No. 08-067) was approved by the Ethics Review Committee for Animal Experimentation of the $\mathrm{Ka}$ wasaki Medical School, Kurashiki, Japan. Male Wistar rats, purchased from Charles River Japan (Kanagawa, Japan), were housed in a temperature- and humidity-controlled room with a 12-hour light-dark cycle; they were fed standard laboratory animal chow and had free access to tap water. Seven-week-old Wistar rats (body weight 200-230 g) were randomly divided into the following three groups ( $\mathrm{n}=8$ in each group): castor oil and ethanol (vehicle for FK506; control group), FK506 at $0.6 \mathrm{mg} / \mathrm{kg} /$ day (FK506 group), and FK506 plus telmisartan at $1 \mathrm{mg} / \mathrm{kg} / \mathrm{day}$ (FK506 + Telm group). The dosage of $0.6 \mathrm{mg} / \mathrm{kg} /$ day FK506 was determined by prelimi- nary experiments. This dosage does not induce diarrhea or hypertension in rats. The dosage of $1.0 \mathrm{mg} / \mathrm{kg} /$ day telmisartan was also determined by preliminary experiments. During the experimental period, body weight was measured weekly. Systolic arterial blood pressure was also measured weekly using the tail-cuff method at an ambient temperature of $37^{\circ} \mathrm{C}$ (BP-98A; Softron, Tokyo, Japan) [16]. The rats were subjected to a 24 -hour fast in metabolic cages to collect urine samples on the day before they were killed. After 9 weeks of treatment, the rats were killed and blood samples were obtained using an 18-gauge needle inserted into the left ventricle. Urinary protein excretion and levels of serum and urinary creatinine were measured. The abdominal aorta was cannulated, and the kidney was perfused in a retrograde manner with ice-cold phosphate-buffered saline (PBS; pH 7.4). One-quarter of each removed kidney was immersed and fixed in $4 \%$ paraformaldehyde and then embedded in paraffin. The remainder of each kidney was cut into small pieces, and glomeruli were isolated by mechanical graded sieving [17] for superoxide production assay, NAD(P)H oxidase activity assay, and mRNA extraction.

\section{Histological Examination}

Sections (4- $\mu \mathrm{m}$ thick) were prepared from renal tissue samples embedded in paraffin and stained with periodic acid-Schiff (PAS) and Masson's trichrome. Kidney sections were photographed and digitized into color images using a Nikon Coolscope (Nikon, Tokyo, Japan).

\section{Detection of Superoxide in Glomeruli}

Superoxide production was detected by $2^{\prime}, 7^{\prime}$-dichlorofluorescein (DCF) staining [18]. Isolated glomeruli from each group were incubated with RPMI-1640 containing $20 \mathrm{M} 2^{\prime}, 7^{\prime}$ dichlorofluorescein diacetate (DCFH-DA; Molecular Probes, Eugene, Oreg., USA) for $10 \mathrm{~min}$ and then rinsed with PBS. Fluorescence images were obtained using a confocal laser microscope (TCS SP2 AOBS MP; Leica Microsystems, Tokyo, Japan) at excitation/emission wavelengths of 485/535 nm for DCF. The fluorescence intensity values from 20 different isolated glomeruli were calculated by Leica TCS-NT software (Leica Microsystems) and presented as average values.

\section{Detection of ED-1-Positive Cells in Glomeruli}

Macrophages were detected by ED-1 staining. Paraffin-embedded kidney sections approximately $4-\mu \mathrm{m}$ thick were deparaffinized. A rat anti-ED-1 monoclonal antibody (Serotec, Oxford, UK) was used as the primary antibody. Antibody binding was detected using a Histofine Simple Stain MAX-PO (MULTI) kit (Nichirei, Tokyo, Japan) and 3,3'-diaminobenzidine (Sigma-Aldrich, St. Louis, Mo., USA). We examined 30 glomeruli of each rat (240 glomeruli per group) and counted ED-1-positive cells under a light microscope. The average number of ED-1-positive cells per glomerulus was compared.

\section{Effect of $N A D(P) H$ Oxidase Activity Induced by FK506}

We used human glomerular endothelial cells (hGECs), passage 8-10 (Cell Systems Corp., Kirkland, Wash., USA). Cells were cultured on 10-cm dishes and propagated in Dulbecco's modified Eagle's medium supplemented with $5 \%$ heat-inactivated newborn bovine serum, $100 \mathrm{U} / \mathrm{ml}$ penicillin, and $100 \mu \mathrm{g} / \mathrm{ml}$ streptomycin at $37^{\circ} \mathrm{C}$ in $\mathrm{CO} 2$. The hGECs were incubated with: castor oil and ethanol (vehicle for FK506); FK506 $\left(10^{-7} \mathrm{M}\right)$; FK506 $\left(10^{-7} \mathrm{M}\right)+$ telmisartan $\left(10^{-6} \mathrm{M}\right.$ or $\left.10^{-5} \mathrm{M}\right)$; or FK506 $\left(10^{-7} \mathrm{M}\right) \pm \operatorname{gp} 91$ tat $(5 \times$ 
$\left.10^{-5} \mathrm{M}\right)$, a NAD $(\mathrm{P}) \mathrm{H}$ oxidase inhibitor [19] for $24 \mathrm{~h}$. In another in vitro experiment, hGECs were incubated with FK506 $\left(10^{-7} \mathrm{M}\right)$ and valsartan $\left(10^{-6}\right.$ or $\left.10^{-5} \mathrm{M}\right)$ or GW9662 $\left(2 \times 10^{-6} \mathrm{M}\right)$, a PPAR- $\gamma$ inhibitor. After incubation, $\mathrm{NAD}(\mathrm{P}) \mathrm{H}$ oxidase activity was measured using a lucigenin chemiluminescence assay.

Lucigenin Chemiluminescence Assay for Measuring $\mathrm{NAD}(\mathrm{P}) \mathrm{H}$ Oxidase Activity in Isolated Glomeruli and hGECs

$\mathrm{NAD}(\mathrm{P}) \mathrm{H}$ oxidase activity in the glomeruli and hGECs was measured in units per minute per milligram using lucigenin chemiluminescence as previously described [20].

RNA Isolation and Real-Time Quantitative PCR

Total RNA was isolated from the glomeruli and hGECs with TRIzol (Invitrogen Japan, Tokyo, Japan). Reverse transcriptase reactions were performed using a Ready-To-Go T-Primed FirstStrand Kit (GE Healthcare Bio-Sciences, Tokyo, Japan) for firststrand cDNA synthesis. Real-time quantitative PCR was performed using the ABI Prism 7700 Sequence Detection System (Applied Biosystems, Foster City, Calif., USA). Primers and probes for TaqMan analysis were designed using Primer Express 1.5 (Applied Biosystems) with information from the supplier based on the sequence information from GenBank or EST databases. We have previously described the primers and probes used for gp9lphox, p22phox, p47phox, p67phox (where phox indicates phagocyte oxidase), and angiotensin II type 1 receptor (AT1R) $[12,17]$. Primer and probe sequences for rat ICAM-1 (NM_012967) were as follows: 5'-GAATCCAGCCCCTAATCTGA-3' (forward primer); 5'-TAAAGGCACGGCACTTGTAG-3' (reverse primer); and 5'-FAMCTGCCTATCGGGATGGTGAAGTCTG-TAMRA-3' (TaqMan probe). Primer and probe sequences for rat VCAM-1 (NM_012889) were as follows: 5'-GGGAAACTGGAAAGAGG-AATC-3' (forward primer); 5'-ATCAGGAGCCAAACACTT-GAC-3' (reverse primer); and 5'-FAM-ATTCAATTCAGTGG-CCCCCTGGA-TAMRA3' (TaqMan probe). Primer and probe sequences for human ICAM1 (NM_000201) were as follows: 5'-CAAGGCCTCAGTCAGTGTGA-3' (forward primer); 5'-CCTC-TGGCTTCGTCAGAATC-3' (reverse primer); and 5'-FAM-TA-CAGCTTTCCGGCGCCCAATAMRA-3' (TaqMan probe). Primer and probe sequences for human VCAM-1 (NM_001078) were as follows; 5'-ATTGGTGACTCCGTCTCATTG-3' (forward primer); $5^{\prime}$-CCTTCCCATTCAGTGGACTATC-3' (reverse primer); and 5'-FAM-CCCCATTTTTCTCTTGGAGAACCCA-TAM-RA-3' (TaqMan probe).

\section{Statistical Analysis}

Values are expressed as means \pm standard error of the mean (SEM). Statistical comparisons were made using the Mann-Whitney $U$ test or the one-factor analysis of variance with a TukeyKramer test for multiple comparisons. A p value of $<0.05$ was considered significant.

\section{Results}

\section{Histological Examination}

Histological analysis was performed on the renal cortex in tissue sections stained with PAS and Masson's trichrome. There were no abnormalities in the glomeruli or tubulointerstitial areas in any groups (data not shown).
Table 1. Physiologic and biochemical data

\begin{tabular}{lccc}
\hline & Control & FK506 & FK506 + Telm \\
\hline Number & 8 & 8 & 8 \\
BW, g & $471 \pm 37$ & $396 \pm 34^{*}$ & $397 \pm 22^{*}$ \\
SBP, mm Hg & $131 \pm 14$ & $121 \pm 8$ & $123 \pm 8$ \\
DBP, mm Hg & $99 \pm 10$ & $98 \pm 12$ & $98 \pm 7$ \\
S-CRN, mg/dl & $0.29 \pm 0.01$ & $0.40 \pm 0.08^{*}$ & $0.33 \pm 0.06^{* *}$ \\
U-PROT/U-CRN & & & \\
$\quad$ mg/g CRN & $0.57 \pm 0.07$ & $0.42 \pm 0.11$ & $0.23 \pm 0.05^{* *}$ \\
\hline
\end{tabular}

$\mathrm{BW}=$ Body weight; $\mathrm{SBP}=$ systolic blood pressure; $\mathrm{DBP}=$ diastolic blood pressure; $\mathrm{S}-\mathrm{CRN}=$ serum creatinine; $\mathrm{U}-\mathrm{PROT}=$ urinary protein excretion; $\mathrm{U}-\mathrm{CRN}=$ urinary creatinine excretion. ${ }^{*} \mathrm{p}<0.05$ vs. Control; ${ }^{* *} \mathrm{p}<0.05$ vs. FK506.

\section{Physiologic and Biochemical Parameters}

Table 1 summarizes the physiological and biochemical parameters in each group. Body weight was significantly decreased in the FK506 and FK506 + Telm groups compared with the control group $(\mathrm{p}<0.05)$. Systolic blood pressure did not differ between groups. Serum creatinine was significantly higher in the FK506 group compared with the other two groups $(\mathrm{p}<0.05)$. There was no difference in urinary protein excretion between the control and the FK506 groups, but this parameter was significantly decreased in the FK506 + Telm group compared with the FK506 group ( $\mathrm{p}<0.05)$.

\section{Glomerular ROS Production}

Oxidation of DCFH to the fluorescent compound DCF was used as a qualitative marker of cellular oxidative stress, because a number of chemicals, such as $\mathrm{H}_{2} \mathrm{O}_{2}$, $\mathrm{ONOO}^{-}$, and $\mathrm{HOCl}$, can produce DCF fluorescence [21]. Figure 1a shows representative glomerular fluorescence images in each group. DCF fluorescence intensity in the isolated glomeruli was significantly stronger in the FK506 group than in the control group, and weaker in the FK506 + Telm group than in the FK506 group. We determined $\mathrm{NAD}(\mathrm{P}) \mathrm{H}$ oxidase activity in isolated glomeruli with a lucigenin chemiluminescence assay (fig. 1b). NAD $(\mathrm{P}) \mathrm{H}$ oxidase activity was also significantly higher in the FK506 group compared with the control group. Increased NAD(P)H oxidase activity induced by FK506 was partially suppressed by telmisartan treatment.

\section{Glomerular mRNA Expression of $N A D(P) H$ Oxidase Components}

p22phox and gp91phox are membrane components of NAD $(\mathrm{P}) \mathrm{H}$ oxidase, whereas $\mathrm{p} 47$ phox and p67phox are cy- 


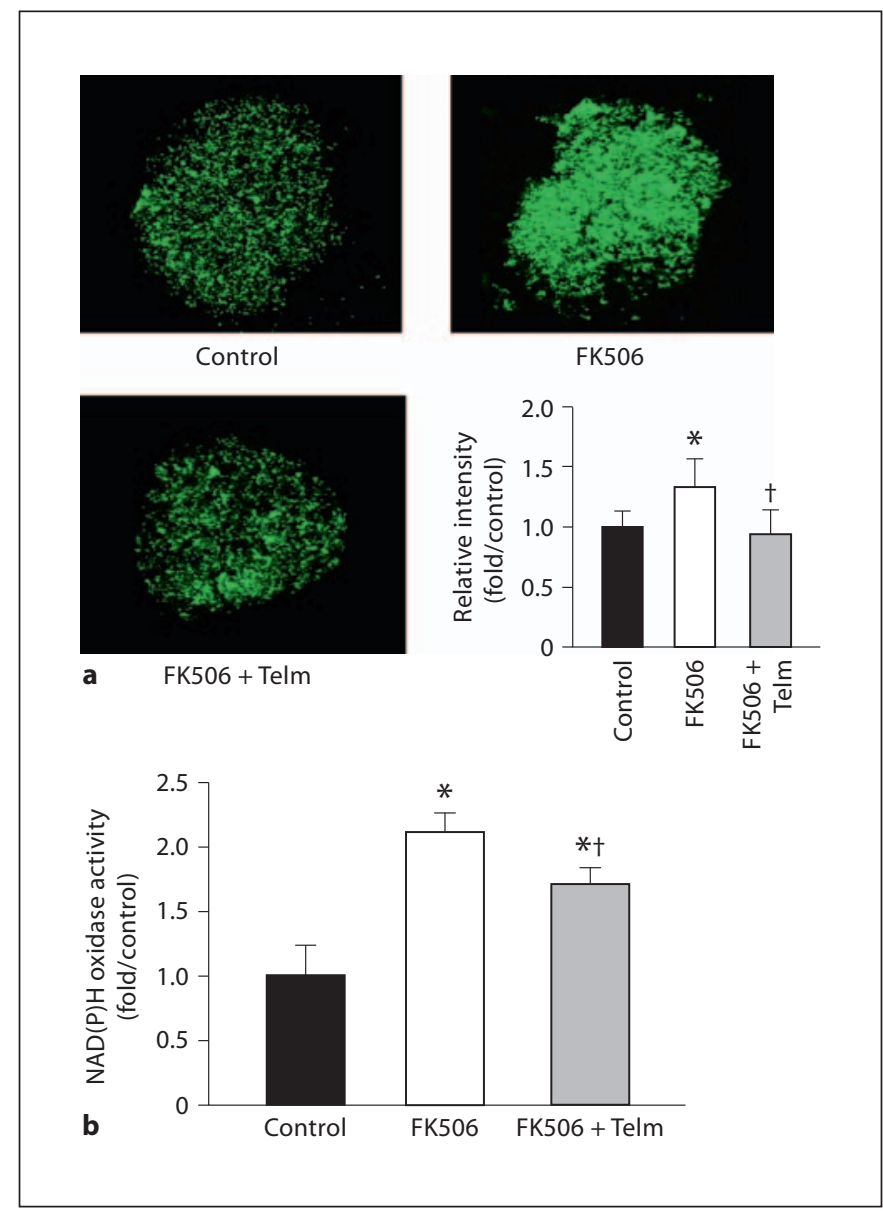

Fig. 1. ROS in the glomerulus. a ROS production detected by DCFH staining in isolated glomeruli. Representative glomerular DCFH staining is shown. b $\mathrm{NAD}(\mathrm{P}) \mathrm{H}$ oxidase activity determined by lucigenin assay. ${ }^{*} \mathrm{p}<0.05$ vs. control; ${ }^{\dagger} \mathrm{p}<0.05$ vs. FK506.

tosolic components. Expression of mRNA of both cytosolic components was significantly increased in isolated glomeruli from the FK506 group. In contrast, expression of mRNA of both membrane components did not significantly differ between the groups (fig. 2a-d).

\section{Glomerular mRNA Expression of Inflammatory \\ Markers}

Expression of ICAM-1 and VCAM-1 mRNA (fig. 3a, b) was increased in the FK506 group compared with the control group. Addition of telmisartan decreased mRNA expression of ICAM-1 and VCAM-1, and ICAM-1 expression was significantly decreased in the FK506 + Telm group compared with the FK506 group.

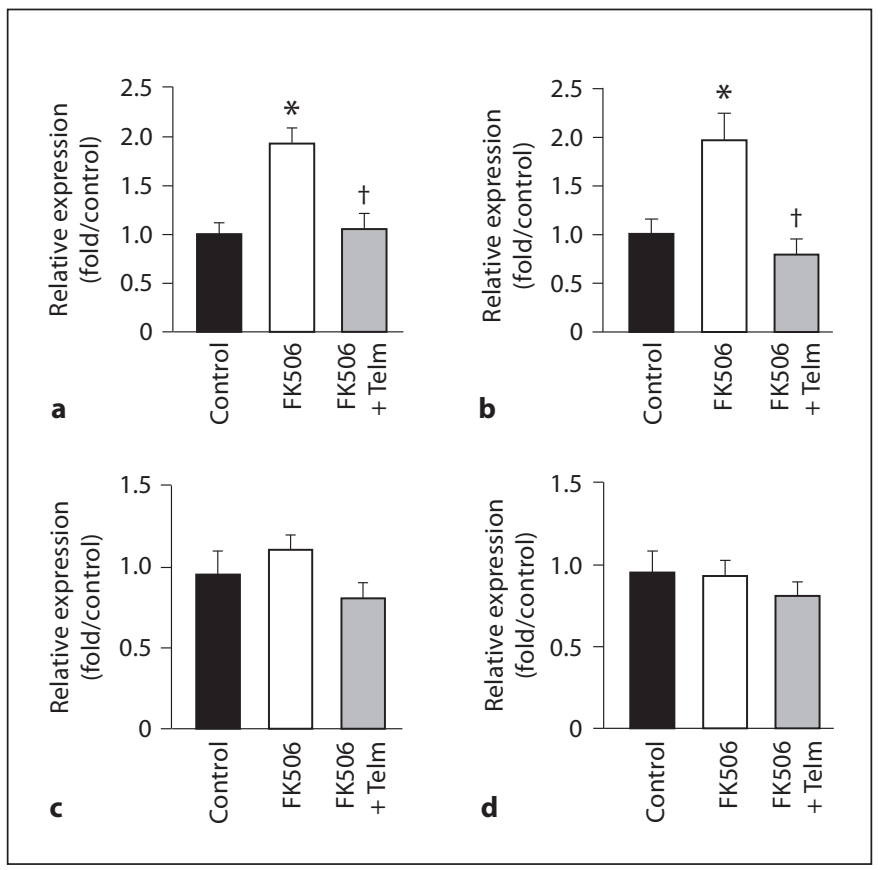

Fig. 2. Glomerular expression of the components of $\mathrm{NAD}(\mathrm{P}) \mathrm{H}$ oxidase. The mRNA levels of cytosolic components p67phox (a) and p47phox (b), and membrane components p22phox (c), and gp91phox (d) were quantitatively analyzed by real-time PCR. ${ }^{*} \mathrm{p}<0.05$ vs. control; ${ }^{\dagger} \mathrm{p}<0.05$ vs. FK506.

\section{Infiltration of ED-1-Positive Cells into Glomeruli}

The number of glomerular ED-1-positive cells was significantly increased in the FK506 group compared with the control group. Addition of telmisartan prevented infiltration of macrophages into glomeruli (fig. 4a, b).

\section{Glomerular mRNA Expression of the Angiotensin}

Type 1 Receptor

We examined the mRNA expression of AT1R in each group. AT1R mRNA expression in isolated glomeruli was significantly increased in the FK506 group compared with the control group. Addition of telmisartan inhibited the expression of AT1R mRNA induced by FK506 (fig. 5).

\section{FK506-Stimulated NAD $(P) H$ Oxidase Activity in hGECs in vitro}

Using hGECs, we investigated the direct effects of FK506 on NAD(P)H oxidase activity in endothelial cells. NAD(P)H oxidase activity in hGECs was significantly increased in the FK506 group, and addition of telmisartan reduced FK506-stimulated $\mathrm{NAD}(\mathrm{P}) \mathrm{H}$ oxidase activity (fig. 6a). Gp91tat almost completely inhibited FK506- 
Fig. 3. Expression of inflammatory markers in the glomerulus. The mRNA levels of ICAM-1 (a) and VCAM-1 (b) were quantitatively analyzed by real-time PCR. * $\mathrm{p}<$ 0.05 vs. control; ${ }^{\dagger} \mathrm{p}<0.05$ vs. FK506.
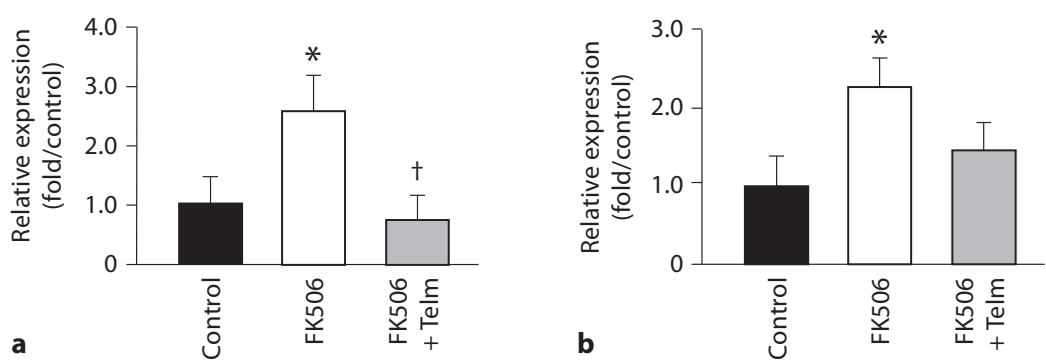

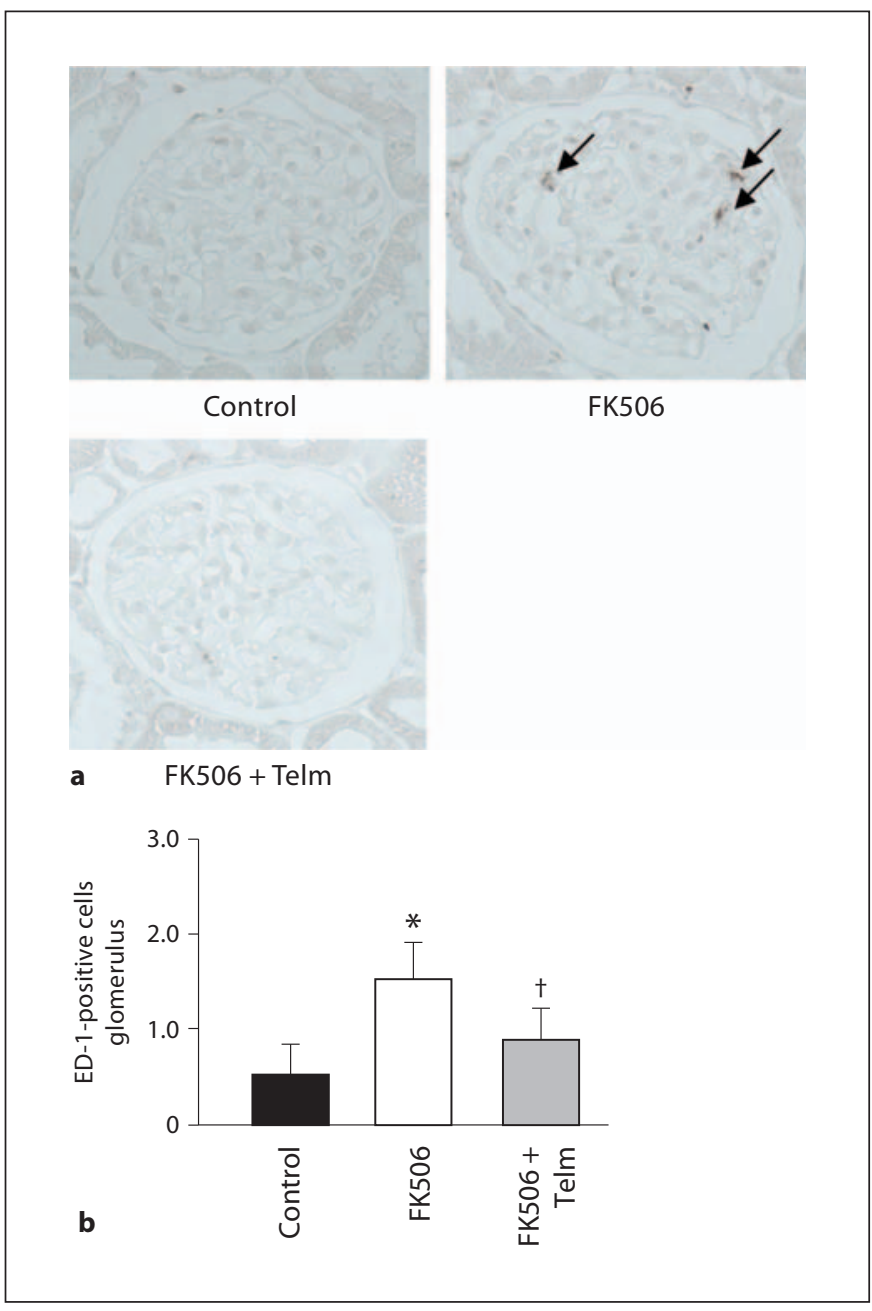

Fig. 4. Macrophage infiltration in the glomerulus. a Immunohistochemical staining of ED-1 (marker for resident macrophages; brown color). Arrows show ED-1-positive cells. b Number of ED1-positive cells per glomerulus. ${ }^{*} \mathrm{p}<0.05$ vs. control; ${ }^{\dagger} \mathrm{p}<0.05$ vs. FK506.

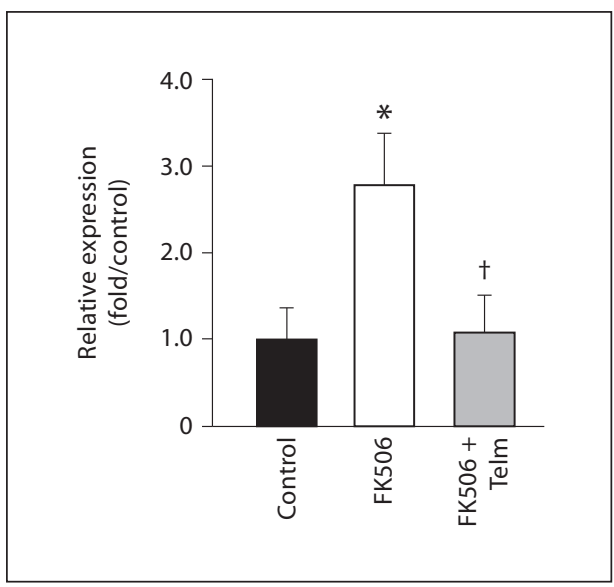

Fig. 5. Angiotensin II type 1 receptor expression in the glomerulus. The mRNA level of angiotensin II type 1 receptor was quantitatively analyzed by real-time PCR. ${ }^{*} \mathrm{p}<0.05$ vs. control; ${ }^{\dagger} \mathrm{p}<$ 0.05 vs. FK506.

stimulated $\mathrm{NAD}(\mathrm{P}) \mathrm{H}$ oxidase activity (fig. 6a), while in contrast to telmisartan, valsartan did not decrease NAD(P)H oxidase activity (fig. 6b). Furthermore, GW9662 abrogated the effects of telmisartan which inhibited NAD(P)H oxidase activity (fig. $6 \mathrm{c}$ ).

\section{FK506-Stimulated ICAM-1 mRNA Expression in hGECs in vitro}

We also investigated the direct effect of FK506 on the expression of ICAM-1 and VCAM-1 mRNA in endothelial cells. ICAM-1 mRNA expression was increased in the FK506 group, and the addition of telmisartan reduced FK506-stimulated ICAM-1 mRNA expression (fig. 6d). The VCAM-1 mRNA expression level in hGECs was too low to detect (data not shown). 


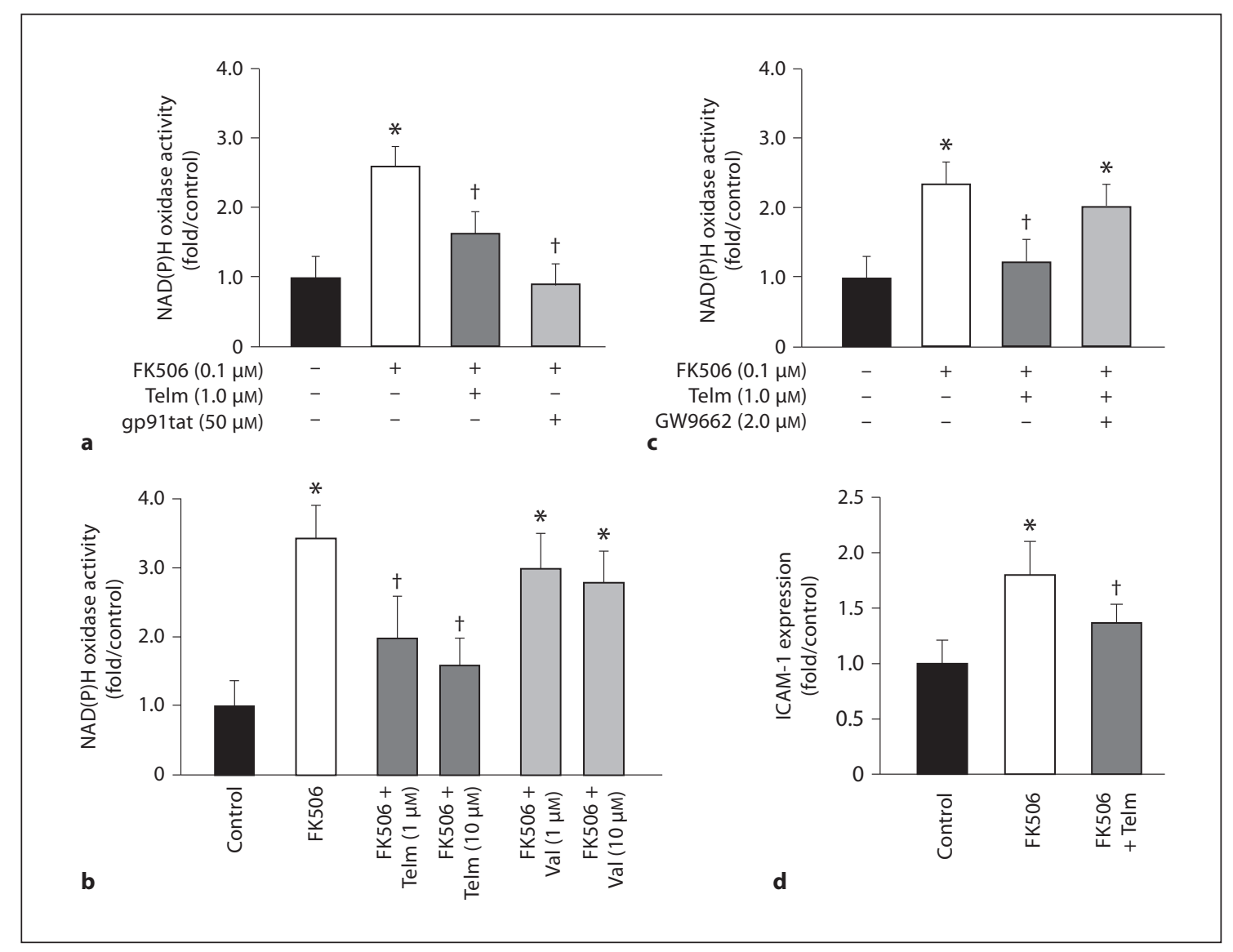

Fig. 6. $\mathrm{NAD}(\mathrm{P}) \mathrm{H}$ oxidase activity and ICAM-1 expression induced by FK506 in human glomerular endothelial cells. a The effect of telmisartan (Telm) or gp91 tat on FK506 induced NAD(P)H oxidase activation. b The effect of Telm or valsartan (Val) on FK506 induced NAD(P)H oxidase activation. c The effect of GW9662 on FK506-induced NAD(P)H oxidase activation; $n=5$ for each experiment. $\mathbf{d}$ The mRNA levels of ICAM-1 by FK506 stimulation and the effect of Telm. ${ }^{*} \mathrm{p}<0.05$ vs. control; ${ }^{\dagger} \mathrm{p}<0.05$ vs. FK506.

\section{Discussion}

The aim of this study was to determine whether FK506 induced glomerular injury by enhancing glomerular oxidative stress. Our findings indicate that FK506 directly induces renal dysfunction and causes glomerular injury via ROS produced by NAD(P)H oxidase. Furthermore, the results of the present study suggest that FK506 induces glomerular inflammatory changes, as glomerular expression of ICAM-1 and VCAM-1 mRNA was upregulated by FK506 which resulted in increased macrophage infiltration. Previous reports [9] have also shown that FK506 increases ROS production and angiotensin II expression. We identified that FK506 increased ROS production in glomeruli, especially in glomerular endothe- lial cells via a direct effect, which is a novel finding. The glomerular injury induced by FK506 was reduced by cotreatment with the angiotensin II receptor blocker telmisartan. These results may lend support to the view that telmisartan exerts a protective effect on the glomerular endothelium.

We have shown that FK506 increases ROS production in the rat glomerulus. Previously, Han et al. [15] reported that FK506 induces ROS production in cultured mesangial cells. In the present study, we have demonstrated that FK506 also induces ROS production in glomerular endothelial cells. The results of several studies have strongly suggested that the production of ROS is a common mechanism of FK506 toxicity. Khanna et al. [22] reported that superoxide mediates renal damage in FK506-induced 
nephrotoxicity in a rat transplant model. Furthermore, Zhou et al. [23] reported that hydrogen peroxide is important for FK506-induced toxicity in renal cells in vitro. These results are compatible with our findings.

In most cases of FK506 nephropathy, increased blood pressure causes adverse effects on renal function. Since our aim was to evaluate the direct effects of FK506 while excluding the effect of hypertension, we used a dosage of FK506 which does not affect blood pressure, and we used a similar dosage of telmisartan. Glomerular injury, such as increased numbers of adhesion molecules and infiltration of macrophages, was observed in FK506 nephropathy. However, there was no significant difference in proteinuria between experimental groups. Most likely, urinary protein which passed through the glomerulus was reabsorbed by the proximal tubule because tubular injury is not severe at the early stage in FK506 nephropathy.

$\mathrm{NAD}(\mathrm{P}) \mathrm{H}$ oxidase is a major source of ROS production in endothelial cells [24]. We have identified that FK506 activates NAD(P)H oxidase activity and increases the $\mathrm{NAD}(\mathrm{P}) \mathrm{H}$ oxidase cytosolic components p47phox and p67phox in the glomerulus. Khanna and Pieper [22] also reported that upregulation of the $\mathrm{NAD}(\mathrm{P}) \mathrm{H}$ oxidase components gp91phox and p22phox was related to production of ROS. In contrast to our results, they reported that membrane components were increased by FK506, but, in agreement with our findings, they showed that $\mathrm{NAD}(\mathrm{P}) \mathrm{H}$ oxidase activity was increased. However, the mechanism of FK506-induced upregulation of NAD $(\mathrm{P}) \mathrm{H}$ oxidase components is not clear and should be explored in future studies. With regard to the mechanism of increased $\mathrm{NAD}(\mathrm{P}) \mathrm{H}$ oxidase activity, there have been some reports of how FK506 might activate enzyme activity. FK506 has been shown to promote dissociation of FK binding protein 12 (FKBP12) from the ryanodine receptor complex and to increase $\mathrm{Ca}^{2+}$ release through ion channels [25]. When FKBP12 is dissociated from the IP3R complex by FK506, calcineurin is also dissociated [26]. Therefore, IP3R is phosphorylated by protein kinase $\mathrm{C}$, and release of $\mathrm{Ca}^{2+}$ through the receptor is increased [27]. These changes in intracellular $\mathrm{Ca}^{2+}$ concentration lead to activation of NAD(P)H oxidase. This mechanism may also be involved in FK506-induced endothelial superoxide production.

There are multiple sources of ROS production, such as COX, eNOS, and mitochondria, with the exception of $\mathrm{NAD}(\mathrm{P}) \mathrm{H}$ oxidase. Gp91tat, a NAD(P)H oxidase inhibitor, almost completely inhibited FK506-stimulated $\mathrm{NAD}(\mathrm{P}) \mathrm{H}$ oxidase activity in vitro. Indeed, FK506-induced ROS production in endothelial cells is mainly a result of $\mathrm{NAD}(\mathrm{P}) \mathrm{H}$ oxidase. We also performed an additional lucigenin assay experiment where we added L-Arg and xanthine as substrates. However, ROS production was not increased in these cases (data not shown). We have considered that $\mathrm{NAD}(\mathrm{P}) \mathrm{H}$ oxidase is a major source of ROS in glomeruli. However, we do not consider that all FK506-induced ROS production in glomeruli is a result of $\mathrm{NAD}(\mathrm{P}) \mathrm{H}$ oxidase because the present study is an in vitro study.

We have shown that FK506 upregulates ICAM-1 and VCAM-1 mRNA expression in the rat glomerulus. Papaccio et al. [28] reported overexpression of adhesion molecules, namely, increased ICAM-1 mRNA in islets, in FK506-treated mice. This finding suggests that FK506 could upregulate expression of adhesion molecules in several tissues. The results of a number of studies have indicated that ROS induce adhesion molecules in endothelial cells. Qin et al. [29] have reported that expression of ICAM-1, VCAM-1, and E-selectin was stimulated through NF- $\mathrm{BB}$ and $\mathrm{p} 38$ MAPK signaling pathways via the induction of ROS. Furthermore, Kim et al. [30] also demonstrated that ICAM-1 and VCAM-1 expression was regulated by ROS-dependent NF- $\kappa \mathrm{B}$ activation. From these reports, it is considered that intraglomerular ROS induced by FK506 resulted in the upregulation of expression of inflammatory adhesion molecules in glomerular endothelial cells.

We have demonstrated that FK506 induced-nephropathy is suppressed by telmisartan treatment. Telmisartan decreased FK506-induced NAD(P)H oxidase activity, ROS production, ICAM1 expression, and macrophage infiltration in glomeruli. Previously, we reported that ARB inhibits ROS production induced by NAD $(\mathrm{P}) \mathrm{H}$ oxidase, and ameliorated endothelial dysfunction in diabetic nephropathy [16]. In the present FK506-induced nephropathy model, the AT1 receptor expression was increased in glomeruli, so it means upregulation of RAS activity. Therefore, the ARB telmisartan shows the protective effects in this FK506 nephropathy model. However, the mechanism of RAS activation by FK506 is not clear at the present time, so it should be clear in the future. On the other hand, the hemodynamic effects of ARB might also be related to protecting against glomerular injury. To evaluate such a postulation, the use of a ROS inhibitor is necessary. This topic warrants further research.

Another possible mechanism of $\mathrm{NAD}(\mathrm{P}) \mathrm{H}$ oxidase activation is the PPAR- $\gamma$-related pathway, in which PPAR- $\gamma$ negatively regulates $\mathrm{NAD}(\mathrm{P}) \mathrm{H}$ oxidase activity [31]. Our findings also suggest that the inhibiting effect of NAD $(\mathrm{P})$ 
$\mathrm{H}$ oxidase activity by telmisartan mediates PPAR- $\gamma$ activation. We previously reported that pioglitazone, a PPAR$\gamma$ agonist, inhibits the expression of the NAD $(\mathrm{P}) \mathrm{H}$ oxidase component and decreases $\mathrm{NAD}(\mathrm{P}) \mathrm{H}$ oxidase activity [32], suggesting that telmisartan has a mechanism similar to that of pioglitazone. Telmisartan has a unique property that activates PPAR- $\gamma$; therefore, it is likely that this activation contributes to its renoprotective effects. Takeda et al. [33] reported that AT1R expression was suppressed by PPAR- $\gamma$ activation. We have shown that glomerular AT1R expression is decreased by telmisartan treatment, and this mechanism of telmisartan may also contribute to RAS inhibition in FK506-treated glomeruli.

\section{Conclusion}

The present findings indicate that oxidative stress induced by FK506 in glomerular endothelial cells is involved mainly in NAD $(\mathrm{P}) \mathrm{H}$ oxidase activity and subsequently induces inflammatory changes. This action could play a role in vascular injury and in complications associated with the long-term use of FK506. In addition, we have shown that telmisartan exerts renoprotective effects against FK506-induced nephrotoxicity via antioxidative activity. In the case of long-term treatment with FK506, we should consider that endothelial dysfunction might develop. Telmisartan might also be effective for endothelial protection from FK506-induced vascular injury.

\section{Acknowledgments}

We thank Ms. Etsuko Yorimasa, Ms. Miki Ishihara, Ms. Satomi Hanada, and Ms. Keiko Ehara (Kawasaki Medical School) for animal care and for help with the in vitro experiments.

\section{Disclosure Statement}

The authors declare no conflicts of interest with regard to the study.

\section{References}

1 Halloran PF: Immunosuppressive drugs for kidney transplantation. N Engl J Med 2004; 351:2715-2729.

2 Henry ML: Cyclosporine and tacrolimus (FK506): a comparison of efficacy and safety profiles. Clin Transplant 1999;13:209-220.

- 3 Tocci MJ, Matkovich DA, Collier KA, Kwok P, Dumont F, Lin S, Degudicibus S, Siekierka JJ, Chin J, Hutchinson NI: The immunosuppressant FK506 selectively inhibits expression of early T cell activation genes. J Immunol 1989;143:718-726.

-4 Dieperink H, Starklint H, Leyssac PP, Kemp E: Glomerulotubular function in cyclosporine-treated rats. A lithium clearance, occlusion time/transit time and micropuncture study. Proc Eur Dial Transplant Assoc Eur Ren Assoc 1985;21:853-859.

5 Williams D, Haragsim L: Calcineurin nephrotoxicity. Adv Chronic Kidney Dis 2006;13: 47-55.

6 Myers BD, Ross J, Newton L, Luetscher J, Perlroth M: Cyclosporine-associated chronic nephropathy. N Engl J Med 1984;311:699_ 705 .

7 Kurtz A, Della Bruna R, Kuhn K: Cyclosporine $A$ enhances renin secretion and production in isolated juxtaglomerular cells. Kidney Int 1988;33:947-953.

8 Hocherl K, Dreher F, Vitzthum H, Kohler J, Kurtz A: Cyclosporine A suppresses cyclooxygenase-2 expression in the rat kidney. J Am Soc Nephrol 2002;13:2427-2436.
9 Cook LG, Chiasson VL, Long C, Wu GY, Mitchell BM: Tacrolimus reduces nitric oxide synthase function by binding to FKBP rather than by its calcineurin effect. Kidney Int 2009;75:719-726.

10 Zhong Z, Arteel GE, Connor HD, Yin M, Frankenberg MV, Stachlewitz RF, Raleigh JA, Mason RP, Thurman RG: Cyclosporin A increases hypoxia and free radical production in rat kidneys: prevention by dietary glycine. Am J Physiol 1998;275:F595-F604.

-11 Diederich D, Skopec J, Diederich A, Dai FX: Cyclosporine produces endothelial dysfunction by increased production of superoxide. Hypertension 1994;23:957-961.

12 Satoh M, Fujimoto S, Haruna Y, Arakawa S, Horike H, Komai N, Sasaki T, Tsujioka K, Makino H, Kashihara N: NAD(P)H oxidase and uncoupled nitric oxide synthase are major sources of glomerular superoxide in rats with experimental diabetic nephropathy. Am J Physiol Renal Physiol 2005;288:F1144-F1152.

13 Fujimoto S, Satoh M, Horike H, Hatta H, Haruna Y, Kobayashi S, Namikoshi T, Arakawa S, Tomita N, Kashihara N: Olmesartan ameliorates progressive glomerular injury in subtotal nephrectomized rats through suppression of superoxide production. Hypertens Res 2008;31:305-313.

14 Vetter M, Chen ZJ, Chang GD, Che D, Liu S, Chang $\mathrm{CH}$ : Cyclosporin A disrupts bradykinin signaling through superoxide. Hypertension 2003;41:1136-1142.
15 Han SY, Mun KC, Choi HJ, Kwak CS, Bae JH, Suh SI, Park SB, Kim HC, Chang EJ: Effects of cyclosporine and tacrolimus on the oxidative stress in cultured mesangial cells. Transplant Proc 2006;38:2240-2241.

16 Kuwabara A, Satoh M, Tomita N, Sasaki T, Kashihara N: Deterioration of glomerular endothelial surface layer induced by oxidative stress is implicated in altered permeability of macromolecules in Zucker fatty rats. Diabetologia 2010;53:2056-2065.

17 Nagasu H, Satoh M, Kuwabara A, Yorimitsu D, Sakuta T, Tomita N, Kashihara N: Renal denervation reduces glomerular injury by suppressing nad(p)h oxidase activity in Dahl salt-sensitive rats. Nephrol Dial Transplant 2010;25:2889-2898.

18 Nagasu H, Satoh M, Yorimitsu D, Tomita N, Sasaki T, Kashihara N: Comparison of combination therapy of olmesartan plus azelnidipine or hydrochlorothiazide on renal and vascular damage in SHR/NDmcr-cp rats. Kidney Blood Press Res 2011;34:87-96.

19 Nagasu H, Satoh M, Kuwabara A, Yorimitsu D, Kidokoro K, Nishi Y, Tomita N, Sasaki T, Kashihara N: Overexpression of klotho protein modulates uninephrectomy-induced compensatory renal hypertrophy by suppressing IGF-I signals. Biochem Biophys Res Commun 2011;407:39-43. 
20 Satoh M, Ogita H, Takeshita K, Mukai Y, Kwiatkowski DJ, Liao JK: Requirement of Racl in the development of cardiac hypertrophy. Proc Natl Acad Sci USA 2006;103: 7432-7437.

21 Onozato ML, Tojo A, Goto A, Fujita T, Wilcox CS: Oxidative stress and nitric oxide synthase in rat diabetic nephropathy: Effects of ACEI and ARB. Kidney Int 2002;61:186-194.

22 Khanna AK, Pieper GM: NADPH oxidase subunits (NOX-1, p22phox, Rac-1) and tacrolimus-induced nephrotoxicity in a rat renal transplant model. Nephrol Dial Transplant 2007;22:376-385.

23 Zhou X, Yang G, Davis CA, Doi SQ, Hirszel P, Wingo CS, Agarwal A: Hydrogen peroxide mediates FK506-induced cytotoxicity in renal cells. Kidney Int 2004;65:139-147.

-24 Guzik TJ, Mussa S, Gastaldi D, Sadowski J, Ratnatunga C, Pillai R, Channon KM: Mechanisms of increased vascular superoxide production in human diabetes mellitus: role of $\mathrm{NAD}(\mathrm{P}) \mathrm{H}$ oxidase and endothelial nitric oxide synthase. Circulation 2002;105:16561662.
25 Timerman AP, Ogunbumni E, Freund E, Wiederrecht G, Marks AR, Fleischer S: The calcium release channel of sarcoplasmic reticulum is modulated by FK-506-binding protein. Dissociation and reconstitution of FKBP-12 to the calcium release channel of skeletal muscle sarcoplasmic reticulum. J Biol Chem 1993;268:22992-22999.

26 Cameron AM, Steiner JP, Roskams AJ, Ali SM, Ronnett GV, Snyder SH: Calcineurin associated with the inositol 1,4,5-trisphosphate receptor-FKBP12 complex modulates $\mathrm{CA}^{2+}$ flux. Cell 1995;83:463-472.

27 Cameron AM, Steiner JP, Sabatini DM, Kaplin AI, Walensky LD, Snyder SH: Immunophilin FK506 binding protein associated with inositol 1,4,5-trisphosphate receptor modulates calcium flux. Proc Natl Acad Sci USA 1995;92:1784-1788.

28 Papaccio G, Latronico MV, Graziano A, Lanza A, Pedulla M: Tacrolimus, but not cyclosporine a, significantly increases expression of ICAM-1 and IFN-gamma in the nod mouse. J Cell Biochem Suppl 2001; (suppl 36):107-116.

29 Qin P, Tang X, Elloso MM, Harnish DC: Bile acids induce adhesion molecule expression in endothelial cells through activation of reactive oxygen species, NF-kappaB, and p38. Am J Physiol Heart Circ Physiol 2006; 291:H741-H747.
30 Kim SR, Bae YH, Bae SK, Choi KS, Yoon KH, Koo TH, Jang HO, Yun I, Kim KW, Kwon YG, Yoo MA, Bae MK: Visfatin enhances ICAM-1 and VCAM-1 expression through ROS-dependent NF-kappaB activation in endothelial cells. Biochim Biophys Acta 2008;1783:886-895.

-31 Toba H, Miki S, Shimizu T, Yoshimura A, Inoue R, Sawai N, Tsukamoto R, Murakami M, Morita Y, Nakayama Y, Kobara M, Nakata T: The direct antioxidative and anti-inflammatory effects of peroxisome proliferator-activated receptors ligands are associated with the inhibition of angiotensin converting enzyme expression in streptozotocin-induced diabetic rat aorta. Eur J Pharmacol 2006;549: 124-132.

32 Namikoshi T, Satoh M, Tomita N, Haruna Y, Kobayashi S, Komai N, Sasaki T, Kashihara $\mathrm{N}$ : Pioglitazone ameliorates endothelial dysfunction in obese rats with nephropathy. Biochem Biophys Res Commun 2007;361: 835-840.

33 Takeda K, Ichiki T, Tokunou T, Funakoshi Y, Iino N, Hirano K, Kanaide H, Takeshita A: Peroxisome proliferator-activated receptor gamma activators downregulate angiotensin II type 1 receptor in vascular smooth muscle cells. Circulation 2000;102:1834-1839. 